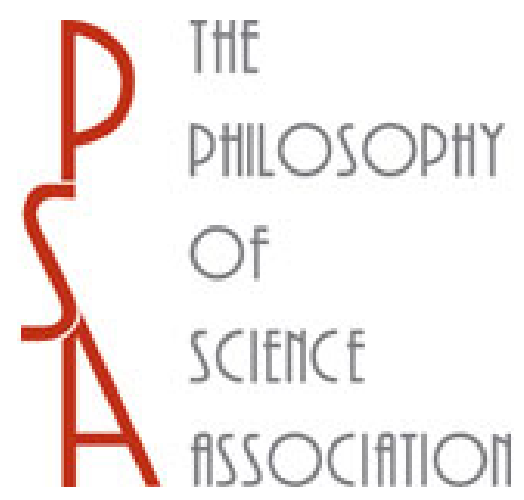

Developmental Systems Theory Formulated as a Claim about Inherited Representations* Author(s): Nicholas Shea

Source: Philosophy of Science, Vol. 78, No. 1 (January 2011), pp. 60-82

Published by: The University of Chicago Press on behalf of the Philosophy of Science Association

Stable URL: http://www.jstor.org/stable/10.1086/658110

Accessed: 08/07/2013 07:31

Your use of the JSTOR archive indicates your acceptance of the Terms \& Conditions of Use, available at http://www.jstor.org/page/info/about/policies/terms.jsp

JSTOR is a not-for-profit service that helps scholars, researchers, and students discover, use, and build upon a wide range of content in a trusted digital archive. We use information technology and tools to increase productivity and facilitate new forms of scholarship. For more information about JSTOR, please contact support@jstor.org. 


\title{
Developmental Systems Theory Formulated as a Claim about Inherited Representations*
}

\author{
Nicholas Shea ${ }^{\dagger+}$
}

Developmental systems theory (DST) is often dismissed on the basis that the causal indispensability of nongenetic factors in evolution and development has long been appreciated. A reformulation makes a more substantive claim: that the special role played by genes is also played by some (but not all) nongenetic resources. That special role can be captured by Shea's 'inherited representation'. Formulating DST as the claim that there are nongenetic inherited representations turns it into a striking, empirically testable hypothesis. DST's characteristic rejection of a gene versus environment dichotomy is preserved but without dissolving into an interactionist casual soup, as some have alleged.

1. Introduction. Developmental systems theory (DST) has a problem. Not one of credibility: DST is taken seriously by many philosophers and endorsed by some. Rather, it faces a problem of perceived inutility. Despite its empirical roots, it motivates relatively little research. That is a loss

*Received October 2009; revised March 2010.

$\dagger$ To contact the author, please write to: Somerville College and Faculty of Philosophy, University of Oxford, 10 Merton Street, Oxford OX1 4JJ, United Kingdom.

$\$$ For helpful comments and discussion of this material, the author would like to thank Paul Griffiths, Arnon Levy, Russell Powell, Ulrich Stegmann, Tobias Uller, the referees for this journal, and audiences in Oxford and at the symposium on Information and Biological Development at the ISHPSSB meeting in Exeter, July 2007. The research reported here was supported by the Oxford University Press John Fell Research Fund, the James Martin 21st Century School, the Wellcome Centre for Neuroethics, and the Mary Somerville Junior Research Fellowship, Somerville College, University of Oxford.

Philosophy of Science, 78 (January 2011) pp. 60-82. 0031-8248/2011/7801-0004\$10.00

Copyright 2011 by the Philosophy of Science Association. All rights reserved. 
because DST carries an important message about the evolutionary significance of nongenetic factors, the truth of which is only now being appreciated more widely. Failing to receive credit where it is due would be merely a pity. Of more consequence is that its perceived lack of utility is continuing to detract from the ability of DST to make its case.

This article argues that one element of the DST canon, its rejection of the notion of genetic information, has been an obstacle to the theory connecting with its target. In arguing that nongenetic factors are critically important, DST has tended to overshoot and suggest that all causal factors are on a par. To make the case against gene centrism, DST should be pointing to the undoubted specialness of genes and saying, "You know that property, the one that makes genes so special? Well that property is found not just in genes but in several other factors in development." That special role is to transmit information, generated through a process of natural selection, down the generations to inform development. This article makes use of my treatment of inherited representations to give an account of that role (Shea 2007).

Relying on a notion of genetic representation will be anathema to many DST theorists (Moss 2001), especially Susan Oyama, who identifies information talk as the source of gene centrism and genetic determinism (Oyama 1985). The claim that genes transmit inherited representations does not have these consequences, so this is intended as a friendly amendment. The research programme of DST can survive even if we do not accept the rejection of the existence of genetic information that is central to DST's philosophy of nature (following a distinction made by GodfreySmith 2001). The aim is to preserve DST's key insight about the developmental and evolutionary importance of nongenetic factors (Griffiths and Gray 1994). That insight is not yet incorporated into the standard orthodoxy of the biological sciences, so there is still work for a reinvigorated DST to do.

Section 2 argues that DST should go for weaker explanatory parity: that some but not all developmental factors are on a par with genes. Section 3 outlines the theory of inherited representations, and section 4 shows how it plausibly applies to a tightly constrained class of nongenetic factors. Section 5 canvasses some rival proposals for narrowing the parity thesis. Finally, section 6 argues that inherited representation is better suited to expressing DST's central hypothesis than are other accounts of genetic information.

2. Explanatory Parity in DST. DST encompasses a variety of views. Our focus is Griffiths and Gray's parity thesis: that genetic and nongenetic factors are on a par in development. This section sets out two versions 
of the parity thesis and commends the more limited form according to which only some other developmental resources are on a par with genes.

Factors other than DNA are clearly causally indispensible in the development of an organism. DNA's initial translation into proteins relies on there already being machinery in the zygotic cell to perform that task. Many other non-DNA factors in the cell are also crucial to an organism's development: DNA methylation patterns and other chromatin marks, basal bodies, microtubule organizing centers, cytoplasmic chemical gradients, and so on.

DST goes further, arguing for the evolutionary importance of developmental factors outside the egg. For example, a supply of vitamin $\mathrm{C}$ is crucial to normal mammalian development. Primates' diet has contained vitamin $\mathrm{C}$ for so long that they have lost the mammalian ability to synthesize it, making primates reliant on vitamin $\mathrm{C}$ in their environment. Hermit crabs' development depends on a ready supply of appropriate empty shells. This too has evolutionary significance. Their use of discarded shells, rather than growing their own, is doubtless a trait that has evolved by natural selection. Furthermore, their fitness depends on which shells are available in the local ecology.

These kinds of examples lead DST to claim explanatory parity between genes and all nongenetic factors: "The full range of developmental resources represents a complex system that is replicated in development" (Griffiths and Gray 1994, 275); "Every element of the developmental matrix which is replicated in each generation and which plays a role in the production of the evolved life-cycle of the organism is inherited" (Griffiths and Gray 1997, 474); "[DST has] a wider conception of the developmental system, not as emerging from interactions between genes, but as emerging from interactions between the whole matrix of resources that are required for development" (Griffiths and Gray 2005, 423). In these passages, DST extends parity very widely. All factors that are causally involved in species-typical development are put on a par. If that is right, DST appears to be saying nothing new. It has long been clear that development is a complex process that depends causally on both genetic and nongenetic factors. No one doubts that organisms would fail to develop normally in the absence of gravity, say (one of DST's examples). DST seems here to be doing no more than emphasizing the causal importance of nongenetic factors in development: each is such that some aspect of the adaptive phenotype would not develop but for it. Indeed, Griffiths and Gray sometimes reject the possibility of making a principled distinction according to which only a subset of other factors is on a par with genes: "There is much to be said about the different roles of particular resources. But there is nothing that divides the resources into two fundamental kinds" (1994, 277). 
In other places, DST theorists adopt a weaker parity thesis, according to which there are many distinctions that can be made between factors found in a developmental system, among which some but not all nongenetic factors are on a par with genes (Griffiths and Knight 1998, 254; the 'more conservative view' mentioned in Griffiths [2001], 399). But they do not offer a clear account of what distinguishes the special developmental causes: if not all, which ones are on a par with genes? DST theorists distance themselves from the claim that 'genes are not important', saying that is a (common) misunderstanding of their position (Griffiths and Gray 2005, 420). Equally, they reject the claim that 'all developmental causes are of equal importance' as being a parody of their position (Griffiths and Knight 1998, 254). In this more recent work, Griffiths and his coauthors make clear that the weaker parity thesis best expresses their view. However, to avoid being understood in these ways, DST needs an account of what the importance of genes amounts to, in the DST worldview, and of why some developmental causes are important in the same way. It could then substantiate a bold empirical claim: that there are nongenetic developmental resources that are on a par with genes.

What, though, is the special property of genes that is shared by some, but not all, nongenetic causes in development? The next section argues that it is to carry inherited representations.

3. Inherited Representations. This section outlines the argument from Shea (2007) that genes carry inherited representations. We should clear away a preliminary objection. Information talk in biology has various bad associations. The claim that genes are information carriers is taken by some to imply genetic determinism (cf. Oyama 1985) or that all geneenvironment interactions are additive (cf. Lewontin 1974) or that phenotypes come preformed in the genes.

I do not doubt that talk of genetic information can attract these unfortunate connotations. As Griffiths has observed (2005), genetic determinism is a popular idea that resolutely refuses to die. However, inherited representation' in the theoretical sense developed by Shea (2007) does not have these implications. Genetic representations can be false, and the instructions they carry can go unsatisfied. Genetic representations do not contain preformed phenotypic outcomes, and they do not determine such outcomes. Nor does the existence of genetic representation presuppose that gene-environment interactions are additive. So these motivations for resisting information talk furnish no good reason to reject the existence of inherited representations in the sense offered here.

Griffiths and Gray have a second objection to accounts of genetic information, which is that they fail in the stated aim of capturing a special role played by genes. There is correlational information whenever, for some 
univocal reason, the state of one system changes the probability that some other system instantiates some property. Shannon's treatment emphasizes that the way that the states of two systems correlate depends on which background 'channel conditions' are held fixed (Shannon 1949; Dretske 1981). Genes carry information about genotypes in this sense, if we treat the circumstances of development as channel conditions that are held fixed. However, Griffiths and Gray (1994) are right to observe that we can equally well fix on the genome as a channel condition, against which variations in the developmental environment will also carry Shannon information about phenotypes.

In response, various theorists argued that genes carry information in a stronger sense than mere correlations - that they carry semantic information, with correctness conditions or satisfaction conditions (i.e., that they are representations). Sterelny, Smith, and Dickison (1996) and Maynard Smith (2000) founded that claim on evolutionary functions - on the function of genes to produce the outcomes for which they have been selected. ${ }^{1}$ These proposals still appeared too liberal, attributing semantic information to not only genes but every factor in development that has an evolutionary function. To counter those objections, Shea (2007) argued that a careful application of the framework of teleosemantics to the genome requires more than just evolutionary functions. I differed from earlier authors in taking seriously the need to identify a consumer of the representations carried by the genome (see also Godfrey-Smith 2006).

The teleosemantic framework applies when there is a consumer system that responds to a range of different representations by producing a range of different outputs (Millikan 1984). Teleosemantics naturalizes the intuitive idea that a token is a representation when a system treats it as a proxy for some further fact $\mathrm{C}$ about the world. The system responds to the token in a way that is appropriate, given that $\mathrm{C}$ obtains. A gene or genotype $\mathrm{G}$ is selected because a phenotypic feature $\mathrm{P}$ with which it correlates is conducive to some feature $\mathrm{C}$ of the environment (including existing features of the organism). Gene $\mathrm{G}$ is then transmitted down the generations so that descendant organisms continue to develop phenotype $\mathrm{P}$. Doing so will be adaptive while condition $\mathrm{C}$ continues to obtain. If it were right to characterize the production of $\mathrm{P}$ by the mechanisms of development, on the basis of reacting to $\mathrm{G}$ in the zygote, as the operation of a consumer system that takes $G$ as input, then it would follow from teleosemantics that $\mathrm{G}$ represents that $\mathrm{C}$ obtains.

Is there, then, a consumer for the selected genes that are transmitted to the zygotes of future generations? Is there a system that has the function

1. Jablonka (2002) also made an ingenious appeal to teleofunctions but in the service of indicative rather than imperative contents. 
of responding to a range of different genotypes by producing a range of different outputs? Shea (2007) argues that such a consumer can only be discerned from the perspective of evolutionary time. A single individual has access to just one set of zygotic DNA, but over phylogenetic time within a lineage, development in different individuals encounters a range of different genotypes and produces a range of different adaptive phenotypes in response. Viewed over phylogenetic time, development seems to be acting as a consumer. But is that its function? Does development have the evolutionary function of responding to a range of different genotypes with a range of different phenotypes?

That is a very demanding constraint. Godfrey-Smith (1999) argues that, in addition to functions of particular genes (his option A), DNA in general may have adaptive functions (option B). Particular genes that are the basis of heritable phenotypes, and have been selected, have teleofunctions under option A. But there is also evidence that the DNA system as a whole, with its mechanisms of transcription, translation, and replication, and of proofreading and repair, also has a teleofunction under option B: the function of transmitting phenotypes down the generations. That function is a higher-order or metalevel function, in that it is not the function to produce any particular object-level trait $\mathrm{X}$ but to produce whatever traits have been selected. Developmental resources that have this metafunction are inheritance systems. If the genome and the associated machinery of development is an inheritance system, then development does indeed have the metafunction of responding to a range of different genotypes with a range of different phenotypes. So it would qualify as a genuine consumer system.

It is a substantial, but plausible, empirical commitment of the theory that DNA does have this metafunction. Bergstrom and Rosvall (forthcoming) point out that DNA is extraordinarily good at storing and transmitting an arbitrary sequence since it is inert, structurally stable, and very easy to replicate, and can store a long and indefinitely extensible sequence in a small space. These features do not yet show that DNA has our exotic metafunction because they may just be the cause of the powerful evolution we have on earth, without being the consequence of some earlier evolutionary process. Maynard Smith and Szathmáry (1995) go further, arguing that DNA-based inheritance evolved out of an RNA world because of the increased fidelity with which DNA transmits selected phenotypes down the generations. Furthermore, there are two stronger lines of evidence that do not depend on the origins of DNA being based on selection for the metafunction.

It is likely that the DNA transmission system has been modified in various ways in order to perform the metafunction of transmitting selected phenotypes. First, the mechanisms of DNA proofreading and repair are, 
very plausibly, adaptations for improved transmission fidelity (Alberts et al. 2004, 169-91). They cannot be explained away as adaptations for somatic cell inheritance since they are also found in single-celled organisms. The second line of evidence turns on the degrees of freedom in the code that links nucleotide triplets to amino acids. The pairings we observe today are not based on any chemical specificity between nucleotides and amino acids but just depend on how the mechanisms of translation are set up. It seems that there has been selection on the triplet-amino acid mapping, sometime in the distant evolutionary past (Haig and Hurst 1991; Freeland and Hurst 1998). Out of all the millions of possible tripletamino acid codes, it seems that the code we have is optimized to reduce the impact of common replication errors (as well as translation errors). The most common errors produce amino acids with similar chemical properties, hence, proteins with similar enzymatic activity. Both of these modifications of the DNA-development system suggest that it has evolved to perform the metafunction of transmitting selected phenotypes, irrespective of why it originally came into existence. ${ }^{2}$ An analogy is the way we stick a piece of felt onto a rock to make it function as a good paperweight.

Given the metafunction, teleosemantics shows how zygotic DNA carries representational content. A gene $\mathrm{G}$ is selected for a heritable phenotypic difference $\mathrm{P}$ that it makes (in a certain population, across the range of environments in which it is found). ${ }^{3}$ Once selected, $G$ will then be transmitted to the zygotic DNA of generations of future organisms, which will continue to develop phenotype P. Gene G may subsequently come to be causally involved in very many phenotypic effects, in combination with other genes or features of the environment, but according to the standard modern synthesis there is a fact of the matter about the phenotypic effect $\mathrm{P}$ for which it was originally selected. There is also a fact about the conditions of the selective environment that were conducive to $\mathrm{P}$ (why there was selection for $\mathrm{P}$ ). Only when that condition $\mathrm{C}$ obtains does $\mathrm{P}$ continue to perform its function in an evolutionarily normal way (i.e., in the way that accounts for its having been selected). The obtaining of $\mathrm{C}$ is a success condition for the output $\mathrm{P}$ that the consumer system produces in response to $\mathrm{G}$. It follows that $\mathrm{G}$ has the indicative content $C$ is the

2. There is a legitimate question about how such selection could have happened. Lineage-based selection is a candidate, especially since DNA's metafunction is likely to be evolutionarily ancient, coming before the evolution of multicellularity. An alternative is that the benefits in long-run fitness achieved through improvements in transmission fidelity can be an effective way for machinery with such a metafunction to evolve, if the short-run fitness costs of increased fidelity are zero or very small.

3. The same story applies to asexual organisms but at the level of the whole genome, in virtue of selection between different genomes. 
case. And the consumer must continue to produce $P$ in response to $G$ if it is to perform its function in an evolutionarily normal way. So teleosemantics also ascribes to $\mathrm{G}$ the imperative content develop $P$. The indicative content will be false if the environment changes so that $\mathrm{C}$ no longer obtains, and the imperative content will go unsatisfied if, because of error or subsequent selection, development fails to produce $\mathrm{P}$ in response to $\mathrm{G}$.

One of the starting points for the account of genetic representation in Shea (2007) was Moss's (2001) influential distinction between the gene D (the causal role of a molecular gene in development) and the gene $\mathrm{P}$ (genetic differences that correlate with phenotypic differences). Moss makes a detailed critique of the gene-as-information based on the relative rarity of genes $\mathrm{P}$ for interesting phenotypes (genetic diseases aside) and offers DST the noninformational gene D instead. Shea (2007) argues that genetic representation is based not on current genes $\mathrm{P}$ but on the existence of a gene $\mathrm{P}$ at the time when a phenotype was selected, as required for evolution by natural selection. That opens up a substantial notion of geneas-information that DST can make use of.

In some cases, $\mathrm{G}$ may have been selected because it produced a range of phenotypic effects $(\mathrm{P} 1, \ldots, \mathrm{Pn})$, each being conducive to different features of the selective environment $(\mathrm{Cl}, \ldots ., \mathrm{Cn})$. In that case, we need a more complex sentence to capture the content of $\mathrm{G}$ : $C 1$ and . . and Cn is the case; develop Pl or . . . or Pn. In cases of adaptive plasticity, the different phenotypic effects may be adapted to variable features of environment $(\mathrm{V} 1, \ldots, \mathrm{Vn})$, with development being cued to variations in the environment in some way. If $\mathrm{G}$ is selected for these reasons, it will come to carry the content: VI or. . . or Vn is the case; develop Pl if VI, . . . , Pn if $V n$.

Notice that, even if a gene is selected for a range of phenotypic effects, this is a small subset of the phenotypes in which it is involved causally. Pleiotropy and polygeny are ubiquitous, so most genes will be involved in the production of very many different phenotypes, and most phenotypes depend on the expression of very many different genes. Nevertheless, the evolutionary story about why a given gene was selected involves only a small subset of these effects. In any particular case, it may be very hard to tell why a given gene was selected, but if evolutionary orthodoxy is even roughly true, then there has been cumulative selection with genes each being selected for some relatively small number of phenotypes.

Relatedly, observe that the sense in which genes represent phenotypes does not imply a tight causal connection between genes and phenotypes. Natural selection just requires a gene-phenotype correlation to be heritable. That connection may be completely contingent, being highly dependent on particular features of the environment in which it was selected 
(that happened to be stable enough at the time of selection). It may also depend heavily on the genetic background against which that variation occurred. So when a genotype $\mathrm{G}$ represents a particular phenotype $\mathrm{P}$, it in no way follows that $G$ specifies $P$ or causally determines $P$ in any environments outside the range in which it was selected. Even the language of $\mathrm{G}$ 'programming for' $\mathrm{P}$ suggests some kind of tight causal connection between genotype and phenotype. The notion of inherited representation does not have those consequences.

One of the motivations for DST was Lewontin's criticism of the 'lock and key' model of evolution (Griffiths and Gray 2005, 418). Lewontin $(1982,1983)$ argued that thinking of organisms as being adapted to preexisting niches or available ways of life underestimates the extent to which organisms construct their own niches. Our approach to genetic representation can capture such cases, where the indicative content is some very general fact about the environment, but the imperative content is a rich specification of a particular phenotype. That is a further strand of DST that can be nicely expressed using the concept of inherited representation.

This article aims to show that a teleosemantic account of inherited representation is adequate to the task of expressing the fundamental insight of DST. I do not argue that no other account of genetic representation could do so. I deal with the main rivals in the literature, but it remains open that a different account of genetic representation, grounded, for example, in control theory, may turn out to be adequate to the same task. If I am wrong about DNA's metafunction, then another account of inherited representation would needed if it is to be used to precisify the claims of DST (the unavailability of which might then motivate a strong version of the parity thesis).

4. Inherited Representations in Other Inheritance Systems. My account of inherited representation is defended in detail in Shea (2007), but the outline above is enough to see how it applies to some, but by no means all, other causal factors in development. The crucial issue is whether a developmental resource is part of an inheritance system, that is, whether it has the metafunction of transmitting selected phenotypes down the generations. Only then will it carry inherited representations.

An example illustrates that many functionally important causal factors are excluded. In Drosophila melanogaster, the way the embryo differentiates into different cell types, so as to form the insect's gross morphology, is driven by concentration gradients of various proteins in the embryo. Initially, these come from maternally derived morphogens (mRNA and the proteins themselves) placed directly into the cytoplasm of the zygote. So initial morphological development is not under the causal influence of the zygote's own DNA. Maternally derived morphogens are part of the 
zygote's inheritance in a wide sense but are not heritable. When experimentally induced variations lead to viable phenotypic differences (with the genetic background held fixed), descent stops at the first generation. The morphogens that the first offspring passes to the zygotes it produces depend on DNA expression and are independent of the configuration of morphogens it received from its mother. By contrast, genetic variation does lead to heritable differences in the way morphogens are passed down the generations (and in resulting morphology). These genetic variants are maternal effect mutants: the effect of the genetic variation is first seen in offspring and is then carried down the generations (Weber 2005, chap. 8).

Maternally derived morphogens are causally indispensable to the organism's development. They count as 'inherited' on the wide understanding adopted by DST. ${ }^{4}$ But they are of only very limited significance to evolutionary questions since changes in morphogen gradients are not transmitted far into the future unless those changes are based on genetic changes. They are part of the causal background to the organism's development, along with myriad other crucial causal factors, but their limited evolutionary significance is reflected in the fact that they do not carry inherited representations.

However, inherited representations are likely to be carried by factors other than the genome. Recent years have seen an explosion of research on transgenerational epigenetic inheritance: mechanisms by which the state of activity of genes is passed on to future generations. For example, gene expression is modulated by modifications to the DNA envelope, including through methylating stretches of chromatin. The wealth of evidence summarized in Jablonka and Raz (2009) shows that epigenetic effects are found in almost every form of life and that many are very long lasting. Given their ubiquity, it is very likely that, as with DNA, there has been selection on epigenetic mechanisms like chromatin marking for the way they transmit selected phenotypes down the generations. So it is very plausible that some epigenetic mechanisms form an inheritance system and so carry inherited representations.

Compare cell membrane structures. When the zygote divides to form a multicelled embryo, it needs to synthesize new cell walls. That process crucially depends on the structure of the zygote's own cell wall and associated elements like centrioles and microtubule organizing centers (which were constructed for the zygote by its mother and which it 'inherits' in DST's broad sense). Unlike the morphogen gradients in Drosophila, some changes to cell wall structures may be heritable, giving rise to long-

4. Griffiths (2001) treats as inheritance "any biological mechanism that produces resemblances between parents and offspring" (399-400). 
term differences in generations of descendants. (Such differences can thus be subject to natural selection.) However, it is much less plausible that cell walls have the relevant metafunction. It is unlikely that there has been selection on this system for the way it transmits phenotypes in general down the generations, especially as the range of variation of outcomes is limited. So it is unlikely that cell wall structures are inheritance systems, falling into the class of interesting cases that make DST a thesis with strong empirical bite.

A final example is learning by imitation. Here, plausibly, evolution has invented a new channel or channels of inheritance, but the test is demanding. Very many kinds of socially mediated learning give rise to behavioral traditions, even through low-level social phenomena like local enhancement (Avital and Jablonka 2000). Once there are transmitted heritable differences, natural selection may act to select the fitter variant (Mameli 2004), but there are few examples in other animals of cumulative selection of behavioral phenotypes that build on one another, ${ }^{5}$ without the operation of gene-based selection. That is much more likely to occur if the learning mechanism has been adapted to the function of transmitting selected (behavioral) phenotypes down the generations.

There is some evidence that there is a form of imitative learning, at least in humans, that has been adapted to perform this function (Shea 2009). Humans exhibit 'blind overimitation', copying the details of the way an observed action is performed, even though they can see a more efficient route to the same result. By contrast, chimps go directly for the demonstrated outcome, cutting out unnecessary actions and employing the most efficient means of achieving the reward. There is even evidence for a developmental trajectory in humans, with younger children performing more like chimps and the disposition for blind overimitation emerging only between 3 and 5 years of age (Whiten et al. 2009). At first glance, the chimps have the more rational strategy. If you can see an easier way of reaching the result, why copy additional details of the demonstrator's action sequence? On reflection, though, we can see that the human strategy could be an adaptation for transmission fidelity. It allows behavioral phenotypes to be transmitted down the generations, even if their utility cannot be checked by any individual — which would be useful if the advantages of the behavioral variant are too long term or stochastic to be reliably detectable by an individual (e.g., the long-term benefits of particular food preparation practices). By contrast, chimps appear to be learning for themselves, using observed behavior as a helpful cue. The otherwise-puzzling disposition for blind overimitation together with some

5. See the examples in Avital and Jablonka (2000). 
other features of human imitative learning suggest that it may have been modified in order to perform the function of transmitting selected behavioral phenotypes (Shea 2009). In that case, it is a further inheritance system: an additional channel by which information, generated by a process of selection over many generations, is transmitted to future generations to allow them to produce adaptive phenotypes.

This summary of other putative inheritance systems suggests that the parity thesis is correct, on the weaker reading (Griffiths 2001, 396): a defensible notion of genetic information does also apply to other causal factors in development, like chromatin marking. That was a prescient insight of DST. The theory's only mistake was, at times, to take such examples to motivate an overly inclusive parity thesis. There is no good reason to treat environmental resources like the shells used by hermit crabs, or primates' dietary vitamin $\mathrm{C}$, as carrying information down the generations in any substantive sense. Inherited representation sharply delineates a subclass of resources that have an important explanatory status.

With the recent upwelling of research discovering extragenetic mechanisms of inheritance all over the place, it is easy to forget just how unorthodox the DST message originally was. DST traces its roots back to Lehrman in the 1950s and the developmental psychobiology of Gottlieb in the 1960s (Griffiths and Gray 2005, 418), when extragenetic inheritance was decidedly a minority interest. Gene centrism became, if anything, only more dominant with the success of models of gene-based selection in the 1970s (e.g., of altruism) and the rise of molecular biology in the 1980s. DST theorists faced a formidable task to persuade the biological community that extragenetic inheritance was more than a rare curiosity. In the 10 years between Jablonka and Lamb's books (1995, 2005), the tide began to turn in favor of the prevalence and importance of mechanisms of extragenetic inheritance. That prescient insight should be DST's key message. It is succinctly captured by the claim that, in addition to the genome, there are other developmental factors that carry inherited representations.

\section{Rival Candidates for Narrowing the Parity Thesis.}

5.1. Three Nested Classes. So far, we have seen that inherited representation is eminently suited to formulating DST's thesis about the developmental and evolutionary importance of nongenetic factors. In this section, we argue that inherited representation is the best candidate for formulating DST as a radical empirical hypothesis.

DST is interested in developmental causes that recur in each cycle of development, so it excludes one-off accidents (Griffiths and Gray 1994, 
286). Among such recurring causal factors, we consider three progressive narrowings that form a nested set of subclasses: $\mathrm{C} 1, \mathrm{C} 2$, and $\mathrm{C} 3$.

Some recurring factors are not functional, for example, traits that have evolved by genetic drift. DST could formulate its parity thesis as: genes are on a par with all other developmental factors that have evolutionary functions (class C1). Evolutionary functions are effects that enter into an explanation of the survival and reproduction of the entities that produce those effects. One of Griffiths and Gray's examples (1994) is the way ducklings' preference for the maternal call of their own species depends on hearing their own, different, call while they are still in the egg. It follows that one of the functions of ducklings' prenatal vocalizations is, surprisingly, to produce a preference for the maternal call. Many of DST's favorite examples fall into this category. A mother rat's disposition to lick the genitalia of her male pups has the function of promoting the nerve supply needed for normal penile function. DST also wants to treat as functional the interactions between an organism and the background conditions of its environment: a plant's location on the earth's surface where there is sun, an animal's interaction with gravity while it grows, primates' interaction with vitamin $\mathrm{C}$ in their fruit diet, and hermit crabs' interaction with other species' shells. Griffiths and Gray (1994, 290-92) emphasize that such processes should be given an evolutionary explanation, so they, too, plausibly fall into class $\mathrm{C} 1$.

Pointing out that factors other than genes have evolutionary functions is no kind of revelation. In many places, DST theorists focus on a more interesting fact: that there are developmental factors other than genes that give rise to heritable phenotypes. A genetic change in the germ line, if viable, causes a phenotypic difference in a long lineage of descendants. Recall the morphogen gradients in Drosophila. Changing a morphogen gradient in the zygote can make a viable phenotypic difference in that individual but goes no further. Changing a gene whose expression is responsible for that morphogen causes a phenotypic effect that is transmitted down the generations. When variation in a developmental factor causes phenotypic variation that is heritable, natural selection can act so that the population evolves toward the fitter variant. Evolution crucially depends on the fact that genetic variation produces heritable phenotypic variation.

In more concessive moments, when DST theorists hint that they do not really think that all developmental resources are on a par, they suggest that this is the class of interest. Gray uses heritability as the acid test of whether developmental resources are transmitted extragenetically (2001, sec. 9.7, 194-96). Griffiths lists various factors that are on a par with genes 'on the more conservative view' and characterizes them as follows: "Changes in these other resources can cause heritable variation that ap- 
pears in all the cells descended from that egg cell” $(2001,400)$. That is our second candidate narrowing, $\mathrm{C} 2$ : causes of heritable phenotypic variation. ${ }^{6}$

The examples of class $\mathrm{C} 1$ discussed above were chosen so as to fall outside class C2. Class C2 also excludes some other cases from the DST literature, for example, some varieties of niche construction. A new termite colony gets a head start in life if it can inhabit an existing termite mound, but changes made directly to the mound are not preserved and transmitted down the generations to further colonies in further mounds (as they would be if caused by a genetic difference). Similarly, changes made directly to beaver dams are unlikely to be heritable. Nevertheless, C2 does capture many of DST's most central examples. Male cowbirds have different song dialects in different regions, without any relevant genetic differences. The male learns the regionally appropriate song partly through feedback from females with a preference for the local song. Given this mechanism, changes to the phenotype (the local song dialect) will end up being transmitted down the generations.

It is unclear that there has been selection between the different songs sung in different subpopulations, but where heritable differences do have fitness consequences, selection can act, even if there are no relevant genetic differences (Mameli 2004). Habitat imprinting is the process by which animals prefer to live in the type of habitat in which they grew up. Apparently, when one population of European mistle thrushes imprinted on parkland, it did better than populations imprinted on forest (cited by Immelmann 1975). The parkland population expanded not because of a genetic difference, or because of continuing transfers from forest populations, but because of the increased fitness of those individuals that had acquired the parkland habit from their forebears. Class $\mathrm{C} 2$ also covers socially transmitted food preferences in rats. A disposition to eat what your mother eats will give rise to behavioral traditions, and, depending on the extent of individual learning, selection could act on those differences. Griffiths and Gray argue that cuckoolike brood parasitism in viduine finches shows selection on imprinting-based variants (1994, 28990). Offspring lay their eggs in the nest of the species that brought them up, and there are different subspecies imprinted on parasitizing different host species (although in this case there are genetically based morphological differences as well).

These examples fall into the very broad class of Darwinian processes

6. Once a heritable phenotype has been genetically selected, it may cease to be heritable, both because of subsequent pleiotropy and epistasis or because of changes in the makeup of the population or developmental environment. The selected gene will continue to be a cause of the transmission of the selected phenotype down the generations. 
(Godfrey-Smith 2007), and they are clearly of importance in characterizing the evolutionary dynamics. As with any kind of learning, they change the space of possibilities that lie on accessible evolutionary trajectories. But imprinting is an extremely limited mechanism for passing on adaptive phenotypes. When a population becomes imprinted on a new habitat or resource, the previous phenotype is replaced. No accumulation of adaptations is possible. Most of the pattern can be understood as a consequence of a genetically based adaptation for habitat imprinting. DST's fire ant example is similar. Whether a queen is large and monogynous or small and polygynous depends on a pheromone that is produced by and persists in the respective colonies. Thus, the phenotypic difference is heritable because of an environmental factor, the pheromone, that is transmitted down the generations. But again, this is a very limited system of heredity. Although the pheromonal differences may be used to trigger a variety of changes, there is only a very limited range of variation in the transmitted resource (the pheromone).

In their most concessive moments, some DST theorists seem to be pointing to something much more radical. Gray argues that DST research should "investigate whether there are adaptive mechanisms for passing on extragenetic inheritance" (2001, 202; emphasis added). If there are other mechanisms for passing on selected phenotypes, in addition to genes, then they would show more than the limited selection between exclusive alternatives illustrated by imprinting. They would enable the accumulation of adaptations. This is the most restrictive narrowing, class C3: the class of interest for DST should be mechanisms with the evolutionary function of transmitting selected phenotypes down the generations. Sterelny (2001) argues for a related distinction based on evolvability - as we will see in the next subsection.

5.2. Class C3 versus Inherited Representation. Class C3 is the class of factors that have the metafunction we relied on earlier as underpinning the existence of inherited representations (sec. 3). We saw in section 4 that there are plausible examples of nongenetic factors with this metafunction (chromatin marking, blind overimitation in humans). If the importance of class $\mathrm{C} 3$ is accepted, we are most of the way home. The only remaining question is, what is the benefit of using informational properties to draw the relevant distinction (as we do in sec. 3) - of formulating DST's hypothesis in terms of inherited representations?

We offer three reasons for understanding inheritance systems (class C3) as information channels. The first is that evolution does not just depend on selection and the generation of etiological functions but also requires information generated by selection to be preserved and transmitted over very many generations. The process of differential survival and repro- 
duction - selection - generates information about which phenotypic variants are better suited to the environments in which they exist. Life can only evolve if that information is somehow transmitted to subsequent generations. DST's message about the evolutionary importance of nongenetic factors is at its strongest when we see that factors in class $\mathrm{C} 3$ can solve this informational problem: DNA is a wonderful solution, but there are others too. The $\mathrm{C} 3$ factors are rightly considered to be new information channels in their own right, precisely because the metafunction that is definitive of class $\mathrm{C} 3$ underpins inherited representation.

At one point, Griffiths and Gray appear to accept an informational take on heredity, as one of many distinctions that can be drawn legitimately between different causal factors involved in development. Although sticking to DST's overall claim that no dichotomy between different factors in development should be privileged, Griffiths and Gray accept that a distinction between sample-based and information-based heredity may be useful for some purposes $(2005,420)$.

Second, it is becoming increasingly clear how right DST theorists were about the number of different means by which parents have long-lasting influences on their offspring (Jablonka and Lamb 1995, 2005; and the very many examples in Jablonka and Raz [2009]). Information is useful as a common denominator by which all these different systems of heredity can be compared (Jablonka and Lamb 2007, 382).

The third point relies on the more controversial claim that inherited representations are read in the course of individual development (Shea, "Inherited Representations Are Read in Development," forthcoming). Understanding class C3 in informational terms allows us to see that inheritance channels are one of several different sources of information that inform individual development, alongside detecting adaptively relevant information in the individual's own environment (adaptive phenotypic plasticity) and receiving informational cues from the previous generation (cross-generational phenotypic plasticity). Space permits only an outline of that claim here. The idea is most simply explained using a gene-based example, but exactly the same point carries over to DST's examples of other inheritance systems that have been adapted for transmitting selected phenotypes.

The idea stretches back to the later Lorenz (1965), in which he argued that organisms inherit genetic information about the type of environment they are likely to face and use it to achieve an adaptive match to their environment. The explanandum is the striking fact that developing organisms manage to arrive at phenotypes that match aspects of their environment in functional ways. For example, African horseflies of the genus Tabanidae pupate in muddy pools (Lambourn 1930). The larva constructs a cylinder consisting of two corkscrew spirals drilled in opposite directions 
through the mud before forming its pupa in the middle of the cylinder, bottom center. When the pool evaporates, and the mud dries out and cracks, the cracks pass neatly around the pupa. The spiral burrowing is thought to be an adaptation to avoid the risk of the pupation chamber being split in two by a crack in the surrounding mud. But how does the larva "know"? That is to say, where does the information come from that tells the larva that the pupation chamber it is about to construct is at risk from cracking as the mud dries out? There is a striking match between the larval phenotype (the spiral burrowing behavior) and a property of its future environment (cracking mud). DST counsels that we should view the organism's relation to its environment as just another aspect of the unfolding complex developmental system. That perspective overlooks a pressing explanandum, not about how the relation evolved, to which DST can offer the standard Darwinian answer, but about where the information comes from, in individual development, that allows the organism to develop in a way that so exquisitely matches its environment. That explanandum cries out for an informational explanation.

In some cases, the organism detects the relevant property directly while it is developing (Leimar, Hammerstein, and Van Dooren [2006] model the choice between this and relying on what we have been calling inherited representations). For example, the water flea Daphnia pulex relies on chemical traces of predators in the water to tell it whether to grow an expensive defensive shell. In Tabanidae, natural selection is the more likely culprit. The standard story about selection on randomly produced genetic variants means that the genotype that gives rise to the corkscrew burrowing comes to correlate with a fitness-relevant feature of its phylogenetic environment, namely, that the pupation environment dries out and cracks. That is information in the correlational sense. The role of information in the proximal causal story about fly development is thereby connected with the distal story about the origin of that information in phylogenetic history. So natural selection not only explains the adaptation, in the usual way, it also explains how the developing organism manages to solve an informational problem. The organism narrows its uncertainty about the nature of the environment in which it will develop by relying on a resource - its genotype - that represents a feature of the environment.

Where natural selection generates information in other inheritance systems (sec. 4), that too informs individual development. For example, if there has been natural selection on variations in transmitted chromatin marks, then the selected chromatin mark will carry useful information about the selective environment, which will guide the organism toward developing an environmentally appropriate phenotype. Blind overimitation is another example, although there it is easy to overlook the role of selection in generating the information. At first glance, the imitator seems 
just to be learning from the model. But recall that the imitator may have no idea why the behavioral phenotype is adaptive (e.g., why a particular food preparation practice is beneficial, on average in the long run). There may be no way for an individual to detect the adaptive significance of the behavior, if the feedback is too stochastic or too long term for an individual to keep track of. In such cases, the way the behavior of the model guides the imitator to an adaptive outcome depends on transmission of information that was generated by selection over very many generations in the evolutionary past.

Cross-generational phenotypic plasticity (Sultan 2000) is closer to the within-generation process by which the water flea detects an environmental correlate of predators than to the transgenerational process by which information generated through natural selection is transmitted to future generations. Many parental effects arise because the parent can give its offspring relevant information about its local environment. In plants, parental effects can convey to the developing seedling useful information about the kind of local microclimate is it likely to find itself in and, so, about which morphological variant or life history strategy it ought to adopt (Galloway and Etterson 2007). The stress-related phenotype expressed in mother rats, that causes the offspring in turn to be highly stress reactive (Meaney 2001), is also plausibly a mechanism whereby the mother can pass to her offspring (in a rather unobvious way) adaptively relevant information about the kind of environment they are likely to find themselves in. These are not mechanisms that have been adapted for transmitting selected phenotypes (inheritance systems/class C3). Instead, they are cross-generational signaling mechanisms by which parents signal an adaptively relevant environmental variable and offspring produce an adaptively plastic phenotype in response (Shea, "Two Modes of Transgenerational Information Transmission," forthcoming). They do not carry inherited representations but are examples of a different means by which the developing organism can narrow its uncertainty about its developmental environment.

In summary, the special role of DNA comes out when we ask an informational question. DST's insight is that other developmental factors may play that role too.

6. Other Accounts of Genetic Information. Other accounts of inherited information have been offered. Shea (2007) has arguments that some of these fail on their own terms, which we will not reopen here. Others are perfectly cogent but do not capture the sense in which inheritance systems transmit representations or semantic information. In this section, we argue that those accounts are less well suited than inherited representation to capturing the important claim at the heart of DST. 
Levy (2010) argues that information talk is a useful metaphor, allowing scientists to break down systems into senders and receivers so as to be able to think about them more easily. In Levy's view, the usefulness of such metaphors does not turn on genes really carrying representational content. The metaphor can be applied in very many situations. So it would not help DST in singling out a special class of developmental factors about which to make its claim.

By contrast, Godfrey-Smith $(1999,2000)$ focuses on a very particular property of DNA. He argues that it is appropriate to talk about DNA in informational terms only because of the triplet code, a precise relation of causal specificity between the linear order of triplet codons on the DNA molecule and the linear order of amino acids in the proteins that are expressed. Stegmann (2005) argues that the way that DNA determines protein products, through template-directed synthesis, makes it an instruction to form those products. ${ }^{7}$ Godfrey-Smith and Stegmann both appeal to very specific features of the way DNA actually operates, with little prospect of the same properties being exemplified by any nongenetic factors. Neither account is suited to the role of locating information in nongenetic resources (rather, they found an argument to the falsity of even the weak parity thesis).

Kim Sterelny has been involved in the development of a series of views about the status of genetic information. Sterelny and Kitcher (1988) argued that a molecular gene codes for a trait relative to a standard background of environment and other genes. The problem was to identify the standard in a principled way. Sterelny et al. (1996) relied on teleology, as did Maynard Smith (2000), with genes representing the outcomes that it was their etiological function to produce. As we have seen, very many developmental factors have evolutionary functions, so these accounts overgenerate. Jablonka (2002) relied on evolutionary functions to underpin indicative contents for genes, based on 'interpreting receivers'. While it is not entirely clear what physical system corresponds to the receivers, Jablonka does not have the constraint of the metafunction identified by Shea (2007), so her account may also overgenerate. Nor does it capture a sense in which genes have imperative contents.

Sterelny (2001) took a different tack, focusing not on representation but on what it is to be a replicator, which he identified as the set of

7. Stegmann's 'instructional content' turns on the claim that DNA determines the kind and order of the operations that will be carried out to produce its protein product. However, this determination only occurs when conditions are right and things go well. So Stegmann may need to appeal to teleology (although not teleosemantics) to specify the circumstances in which effects flowing from DNA count as being among the 'determinings' that are relied on in discerning instructional content. 
developmental resources that are like DNA in being highly evolvable. A subsidiary point was that such evolvable systems are unlikely to have arisen by chance but have probably been adapted for the transmission of similarity across the generations - that is, to be in our class of inheritance systems, C3. Sterelny (2001) did much to spell out the conditions that underpin evolvability. Since carrying inherited representations in our sense is based on a mechanism having the metafunction that is evidenced by Sterelny's evolvability criteria, the two accounts will agree about most of the cases - about which factors DST should identify as being on a par with genes from an evolutionary and developmental point of view. My account differs from Sterelny in two respects, one local and one global.

The local difference is that Sterelny does not think that all inheritance systems involve information transfer (2001, 346 and 347). Those which involve transfer of samples from parents to offspring are not cases of information transmission, in his view. Sterelny (2004) develops the distinction, spelling out the distinctive evolutionary characteristics of samplebased inheritance.

In my view, the fact that samples are transferred in a process of inheritance does not exclude the fact that information is also transmittedthat the samples carry representational content (of our 'inherited representation' variety). There is no such exclusion with representation in general. When I ask a baker, "what's good?" he may just pass me a slice of cake. The cake is a sample but is also an answer to the question, carrying a semantic content along the lines of this is good. Similarly, some of the examples of sample-based inheritance discussed by Sterelny $(2001,2004)$ are also cases in which information, generated by a process of selection, is being transmitted to future generations. If they are adapted for the transmission of similarity across generations, as Sterelny suggests (2001, 338 and 346), these mechanisms are thereby carrying inherited representations, for the reasons given in sections 3 and 4 above.

Not all the samples that lead to cross-generational similarity will carry inherited representations since some may be a cause of cross-generational similarity (class $\mathrm{C} 2$ ) without being an adaptation for transmitting crossgenerational similarity ( $\mathrm{C} 3$, which is a proper subset of $\mathrm{C} 2$ ). In an obligate symbiosis, say between an insect species and a particular digestive bacterium, the mechanism for passing on a sample of the bacterium in the insect's eggs does not look to have been designed to carry a range of different messages. Even where there is a range of possible symbionts, as when juvenile ruminants ingest faeces to acquire microorganisms that are needed for digestion, the case is more like that of imprinting. The mechanism can give rise to heritable features, but that is not its purpose. Its evolutionary function is to pass on symbiotic microorganisms. Furthermore, some passing of samples may be cases of cross-generational phe- 
notypic plasticity (Sultan 2000, discussed above). In any event, there does not seem to be anything like the fancy metafunction exemplified by DNA. So, while some cases of sample-based heredity do not count as transmitting semantic information, some do. Transfer of samples does not exclude transfer of semantic information.

So much for the local disagreement with the later Sterelny $(2001,2004)$. The more global disagreement concerns the importance of information. Sterelny identifies the class of systems that should be of interest to DST directly in terms of conditions on evolvability. While this is evidence of class $\mathrm{C} 3$, it is not equivalent. Furthermore, there are explanatory benefits of focusing on the inherited representations that are carried by factors in class $\mathrm{C} 3$. The three reasons discussed in section 5.2 above amount to a strong argument that formulating DST's thesis in informational terms is explanatorily superior and better captures the spirit of the DST project.

7. Conclusion. Developmental systems theory has championed a research programme focused on investigating the developmental and evolutionary importance of factors other than genes. That insight has been vindicated by the recent flowering of empirical work in the field. However, DST's distinctive contribution has not been brought sharply into focus. This article has argued that a detachable part of the DST canon-a philosophy of nature that rejects any appeal to semantic information-has obscured its central insight. DST's claim is not just that nongenetic factors are causally important. Formulated as a claim about inherited representations, DST turns into the striking thesis that some factors other than genes also carry genuinely semantic information, in a restricted and rather special way. If that is right, then there is a strong sense in which there are nongenetic channels of inheritance. The payoff is that we can use information as a common currency to compare inheritance systems and to understand how information generated by the process of natural selection is relied on to guide development toward adaptive phenotypes.

\section{REFERENCES}

Alberts, B., et al. 2004. Essential Cell Biology. 2nd ed. New York: Garland Science.

Avital, Eytan, and Eva Jablonka. 2000. Animal Traditions: Behavioural Inheritance in Evolution. Cambridge: Cambridge University Press.

Bergstrom, Carl T., and Martin Rosvall. Forthcoming. "The Transmission Sense of Information." Biology and Philosophy.

Dretske, Fred. 1981. Knowledge and the Flow of Information. Cambridge, MA: MIT Press.

$\rightarrow$ Freeland, S. J., and L. D. Hurst. 1998. "The Genetic Code Is One in a Million." Journal of Molecular Evolution 47:238-48.

Galloway, L. F., and J. R. Etterson. 2007. "Transgenerational Plasticity Is Adaptive in the Wild." Science 318:1134-36.

Godfrey-Smith, Peter. 1999. "Genes and Codes: Lessons from the Philosophy of Mind." In 
Where Biology Meets Psychology: Philosophical Essays, ed. V. Hardcastle. Cambridge, MA: MIT Press. 44

. 2000. "On the Theoretical Role of "Genetic Coding." Philosophy of Science 67:26-

- 2001. "On the Status and Explanatory Structure of Developmental Systems Theory."

In Cycles of Contingency: Developmental Systems and Evolution, ed. Susan Oyama, Paul

E. Griffiths, and Russell D. Gray. Cambridge, MA: MIT Press.

- 2006. "Information in Biology." In The Cambridge Companion to the Philosophy of Biology, ed. D. Hull. Cambridge: Cambridge University Press.

. 2007. "Conditions for Evolution by Natural Selection." Journal of Philosophy 54 (10): 489-516.

Gray, Russell D. 2001. "Selfish Genes or Developmental Systems?" In Thinking about Evolution: Historical, Philosophical and Political Perspectives, ed. R. S. Singh, B. K. Costas, D. B. Paul, and J. Beatty, 184-207. Cambridge: Cambridge University Press.

$\rightarrow$ Griffiths, Paul E. 2001. "Genetic Information: A Metaphor in Search of a Theory." Philosophy of Science 68:394 412.

- 2005. "The Fearless Vampire Conservator: Philip Kitcher, Genetic Determinism and the Informational Gene." In Genes in Development: Re-reading the Molecular Paradigm, ed. E. M. Neumann-Held and C. Rehmann-Sutter. Durham, NC: Duke University Press.

Griffiths, Paul E., and Russell D. Gray. 1994. "Developmental Systems and Evolutionary Explanations." Journal of Philosophy 91:277-304.

$\rightarrow$. 1997. "Replicator II: Judgement Day." Biology and Philosophy 12:471-92.

2005. "Discussion: Three Ways to Misunderstand Developmental Systems Theory." Biology and Philosophy 20:417-25.

$\rightarrow$ Griffiths, Paul E., and Robin D. Knight. 1998. "What Is the Developmentalist Challenge?" Philosophy of Science 65:253-58.

Haig, D., and L. D. Hurst. 1991. "A Quantitative Measure of Error Minimization in the Genetic Code." Journal of Molecular Evolution 33:412-17.

Immelmann, K. 1975. "Ecological Significance of Imprinting and Early Learning." Annual Review of Ecology and Systematics 6:15-37.

Jablonka, Eva. 2002. "Information: Its Interpretation, Its Inheritance, and Its Sharing." Philosophy of Science 69:578-605.

Jablonka, Eva, and Marion J. Lamb. 1995. Epigenetic Inheritance and Evolution: The Lamarckian Dimension. Oxford: Oxford University Press.

- 2005. Evolution in Four Dimensions: Genetic, Epigenetic, Behavioral, and Symbolic Variation in the History of Life. Cambridge, MA: MIT Press.

_. 2007. "Précis of Evolution in Four Dimensions." Behavioral and Brain Sciences 30: 353-92.

Jablonka, Eva, and Gal Raz. 2009. "Transgenerational Epigenetic Inheritance: Prevalence, Mechanisms, and Implications for the Study of Heredity and Evolution." Quarterly Review of Biology 84 (2): 131-76.

Lambourn, W. A. 1930. "The Remarkable Adaptation by which a Dipterous Pupa (Tabanidae) Is Preserved from the Dangers of Fissures in Drying Mud." Proceedings of the Royal Society of London B 106:83-87.

Leimar, O., P. Hammerstein, and T. J. M. Van Dooren. 2006. "A New Perspective on Developmental Plasticity and the Principles of Adaptive Morph Determination." American Naturalist 167:367-76.

Levy, Arnon. 2010. "Information in Biology: A Fictionalist Account." Nous, doi: 10.1111/ j.1468-0068.2010.00792.x.

Lewontin, Richard C. 1974. "The Analysis of Variance and the Analysis of Causes." American Journal of Human Genetics 26:400-411.

. 1982. "Organism and Environment." In Learning, Development, Culture, ed. H. Plotkin, 151-70. New York: Wiley.

. 1983. "The Organism as the Subject and Object of Evolution." Scientia 118:65-82.

Lorenz, K. 1965. Evolution and Modification of Behavior. Chicago: University of Chicago Press. 
Mameli, Matteo. 2004. "Nongenetic Selection and Nongenetic Inheritance." British Journal for the Philosophy of Science 55:35-71.

Maynard Smith, John. 2000. "The Concept of Information in Biology." Philosophy of Science 67:177-94.

Maynard Smith, John, and Erös Szathmáry. 1995. The Major Transitions in Evolution. Oxford: Freeman.

Meaney, M. J. 2001. "Maternal Care, Gene Expression, and the Transmission of Individual Differences in Stress Reactivity across Generations." Annual Review Neuroscience 24: 1161-92.

Millikan, Ruth Garrett. 1984. Language, Thought and Other Biological Categories. Cambridge, MA: MIT Press.

Moss, L. 2001. "Deconstructing the Gene and Reconstructing Molecular Developmental Systems." In Cycles of Contingency: Developmental Systems and Evolution, ed. Susan Oyama, Paul E. Griffiths, and Russell D. Gray. Cambridge, MA: MIT Press.

Oyama, Susan. 1985. The Ontogeny of Information: Developmental Systems and Evolution. Cambridge: Cambridge University Press.

Shannon, C. E. 1949. "The Mathematical Theory of Communication." In The Mathematical Theory of Communication, ed. C. E. Shannon and W. Weaver. Urbana: University of Illinois Press.

Shea, Nicholas. 2007. "Representation in the Genome, and in Other Inheritance Systems." Biology and Philosophy 22:313-31.

$\rightarrow$. 2009. "Imitation as an Inheritance System." Philosophical Transactions of the Royal Society B 364:2429-43.

- Forthcoming. "Inherited Representations Are Read in Development." British Journal for the Philosophy of Science.

- Forthcoming. "Two Modes of Transgenerational Information Transmission." In Signaling, Commitment, and Emotion, ed. Brett Calcott, Richard Joyce, and Kim Sterenly. Cambridge, MA: MIT Press.

Stegmann, Ulrich E. 2005. "Genetic Information as Instructional Content." Philosophy of Science 72 (3): 425-43.

Sterelny, Kim. 2001. "Niche Construction, Developmental Systems and the Extended Replicator." In Cycles of Contingency: Developmental Systems and Evolution, ed. Susan Oyama, Paul E. Griffiths, and Russell D. Gray, 333-50. Cambridge, MA: MIT Press.

- 2004. "Sybiosis, Evolvability and Modularity." In Modularity in Development and Evolution, ed. Gerhard Schlosser and Günter Wagner. Chicago: University of Chicago Press.

Sterelny, Kim, and Philip Kitcher. 1988. "The Return of the Gene.” Journal of Philosophy 85:339-61.

Sterelny, Kim, K. C. Smith, and M. Dickison. 1996. “The Extended Replicator.” Biology and Philosophy 11:377-403.

Sultan, S. E. 2000. "Phenotypic Plasticity for Plant Development, Function and Life History." Trends in Plant Science 5:537-42.

Weber, M. 2005. Philosophy of Experimental Biology. Cambridge: Cambridge University Press.

Whiten, Andrew, N. McGuigan, S. Marshall-Pescini, and L. M. Hopper. 2009. "Emulation, Imitation, Overimitation and the Scope of Culture for Child and Chimpanzee." Philosophical Transactions of the Royal Society B 364:2417-28. 